\title{
Zellulare Transportfahrzeuge für flexible und wandelbare Intralogistiksysteme
}

\author{
Cellular Transport Vehicles for Flexible and Changeable \\ Facility Logistics Systems
}

\author{
Thomas Kirks * \\ Jonas Stenzel* \\ Andreas Kamagaew* \\ Michael ten Hompel ** \\ * Fraunhofer-Institut für Materialfluss und Logistik IML, Dortmund \\ ** Technische Universität Dortmund, Fakultät Maschinenbau, Lehrstuhl für Förder und Lagerwesen, Dortmund
}

A ufgrund stetig wechselnder Leistungsanforderungen volatiler Märkte ist die Flexibilisierung des innerbetrieblichen Materialflusses eine Notwendigkeit. Klassische Fördermittel, wie zentral gesteuerte Stetigfördertechniksysteme, stoßen dabei an ihre Grenzen. Auch die planerische Auslegung von starren Materialflusssystemen ist damit vor eine nicht lösbare Aufgabe gestellt. Zellulare Intralogistiksysteme sind die heutigen Antworten auf diese Anforderungen. Die Zellularen Transportfahrzeuge „Multishuttle Move“ sollen Stetigfördersysteme und manuelle Transporte dort ablösen, wo ein hohes Maß an Flexibilität und Wandelbarkeit gefragt ist, die Planungssicherheit nicht gewährleistet ist oder aufgrund mangelnder Flexibilität nicht automatisiert werden kann. In diesem Beitrag werden diese Systeme vorgestellt und deren Eigenschaften aufgezeigt.

[Schlüsselwörter: Zellulare Intralogistik, Zellulare Transportfahrzeuge, Internet der Dinge, Flexibilität, Wandelbarkeit]

D ue to steadily changing performance requirements of volatile markets a flexibilization of inhouse material flow becomes a necessity. Classical conveyor systems like centrally controlled steady conveyor systems reach their functional limits. In addition, methods of planning of steady material flow systems encounter unsolveable tasks. The answers to these questions offered cellular transport systems. The Cellular Transport Vehicle "Multishuttle Move” shall replace steady conveyor systems and manual transports, where a high degree of flexibility and changeablity is demanded, planning reliability is not guaranteed or automation is not applicable because of lacking flexibility. This paper presents an introduction to these systems and points out their features.

[Keywords: cellular intra-logistics, cellular transport vehicles, internet of things, flexibility, changeability]

\section{EINLEITUNG}

Stetig wechselnde Anforderungen hochvolatiler Märkte stellen heute den Logistiker vor kaum lösbare Aufgaben. Aktuelle Automatisierungskonzepte können diesen schwer gerecht werden, da sie nach dem Stand heutiger Technik entweder unflexibel oder teuer sind. Erweiterungen intralogistischer Systeme sind aktuell mit hohen Kosten für Anpassungen und Softwareänderungen verbunden. Neue Systeme sollten schnell und flexibel auf stetig wechselnde Leistungsanforderungen reagieren und kostengünstig skalierbar sein. Dabei bieten sich Lösungswege an, die schon aus dem Consumer-Markt bekannt sind: „plug and convey“ als Pendant zu „plug and play“[GtH10; SRS07].

\section{FORSCHUNGSAKTIVITÄTEN}

Die Interdisziplinarität der Intralogistik bietet Einblicke in unterschiedliche Domänen wie angewandte Informatik, Mathematik, Steuerungs- und Regelungstechnik, Robotik sowie weitere Ingenieursdisziplinen. Zunächst werden aktuelle Entwicklungen in der Intralogistik mit dem Schwerpunkt Zellularer Transportsysteme genannt. Anschließend wird auf die Forschungsschwerpunkte des Fraunhofer IML, das sich mit der Entwicklung des „Multishuttle Move“-Systems (MS Move) auseinandersetzt, eingegangen.

\subsection{ANGEWANDTE ZELLULARE INTRALOGISTIK}

Aktuell existieren einige Realisierungen, die sich der Zellularen Intralogistik zuordnen lassen. Dabei werden statische Einrichtungen, wie Stetigfördertechniksysteme durch Transportfahrzeuge ersetzt. Bekannte Realisierungen davon sind: 


\subsubsection{KIVA SYSTEMS}

Das KIVA-System besteht aus einigen wenigen oder bis zu hunderten von Robotern, die in der Lage sind Regaleinheiten anzuheben und sie in einem Lager zu transportieren [Gui08]. Die derzeit größte Implementierung besteht aus 500 Einheiten, die Güter in einem Warenlager von STAPLES, U.S.A. bewegen [AWM08] (vgl. Abbildung 1). Die Roboter, genannt „driving pods“, verwenden zwei Kameras, die aus Barcodes bestehende künstliche Landmarken auf dem Boden und unter den Regal zur Navigation erfassen. Diese Informationen werden mit Messwerten von anderen Sensoren wie Encodern, Beschleunigungssensoren und Gyroskopen kombiniert. Die Roboter verwenden eine rasterbasierte Streckennavigation. Sie können nicht gleichzeitig rotatorische und translatorische Bewegungen ausführen. Die Maximalgeschwindigkeit ist auf $1,3 \mathrm{~m} / \mathrm{s}$ begrenzt [AW08]. Der Fokus des Projektes lag auf der Entwicklung eines zentralgesteuerten MultiAgenten-Systems zur Steuerung der Roboter.



Abbildung 1. KIVA-System im Lager

\subsubsection{KARIS PROJECT}

Mit dem KARIS-Projekt wird am Karlsruher Institut für Technik (KIT) ein flexibles Materialflusssystem entwickelt [HFS09; May09]. Das System besteht aus mehreren autonomen Fahrzeugen, mit je einer Größe von einem halben Quadratmeter, bei einer Höhe von $40 \mathrm{~cm}$ und einem aufgebauten Rollenförderer (siehe Abbildung 2). Diese Fahrzeuge sind in der Lage sich an sich ändernde Bedingungen anzupassen und sich sowohl in einem Verbund zusammenzuschließen um schwere Lasten zu tragen als auch eine konventionelle Förderstrecke für hohen Durchsatz zu bilden. Zu diesem Zweck können sie sich in einer bekannten Umgebung mit Laserscannern lokalisieren und über WiFi untereinander kommunizieren.

\subsubsection{ADAM AGV}

ADAM ist ein Fahrerloses Transportfahrzeug (FTF) des Herstellers RMTRobotics in Zusammenarbeit mit Mobile Robots [RMT07]. Es verwendet eine freie Navigation mit der Software „MobilePlanner“ von Mobile Robots. Die Maximalgeschwindigkeit beträgt 1,5 m/s. Das
ADAM-System realisiert eine modulare Fahrzeugplattform, stellt jedoch kein Komplettsystem dar und besitzt kein Lastaufnahmemittel (siehe Abbildung 3). Bis zu 5 ADAM-Roboter bilden einen Verbund, der als „Flotte“ bezeichnet wird. Eine Flotte wird mit einem PCbasierten Steuerungssystem kontrolliert. Interaktion zwischen den Einheiten ist nicht vorgesehen.

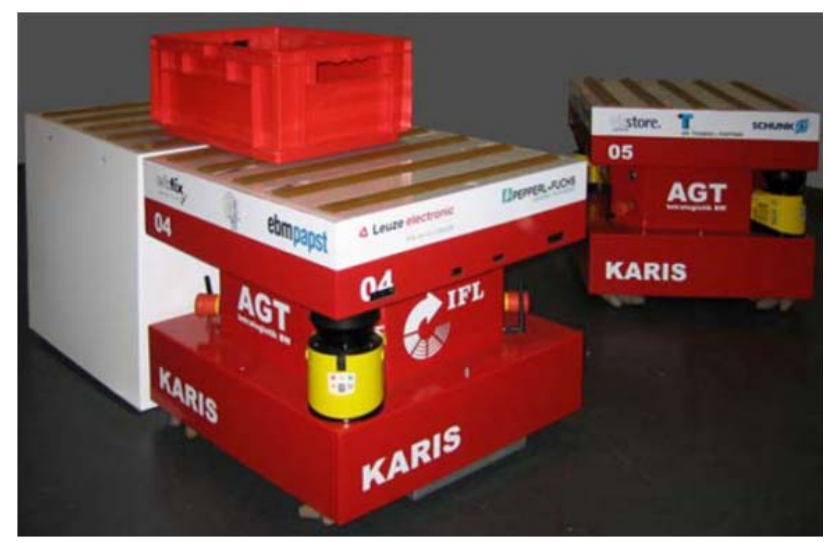

Abbildung 2. KARIS-System Fahrzeug

\subsubsection{ARMADA}

Das „Basic Intralogistic Element“ (BInE) repräsentiert ein autonomes Modul, welches in der Lage ist kleine Behälter sowie eine Palette als Zusammenschluss mehrerer BInEs zu transportieren [BSS08] (siehe Abbildung 4). Durch einen aufgesetzten bidirektionalen Antrieb sind mehrere Module in der Lage einen Stetigförderer zu bilden. Dieses prototypische System wurde im ARMADA (Autonomous Reliable Material Handling System of Aggregated Redundant Distributed Actuators)-Projekt entwickelt. Das Hauptaugenmerk lag auf der mechanischen Konstruktion und der Steuerung der Formation der Module ohne Beachtung von Sensorik, Energieversorgung und Navigationstechnik.

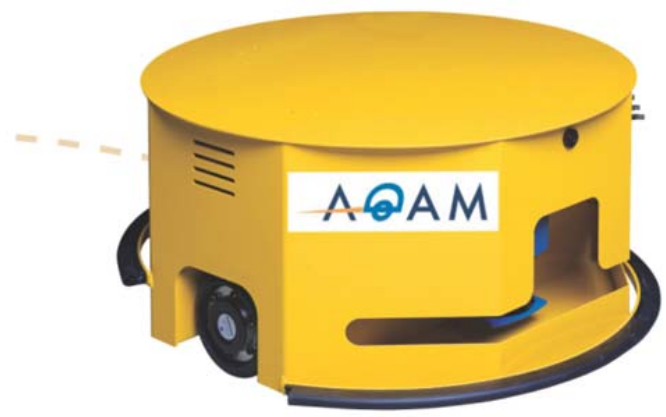

Abbildung 3. ADAM-Plattform 


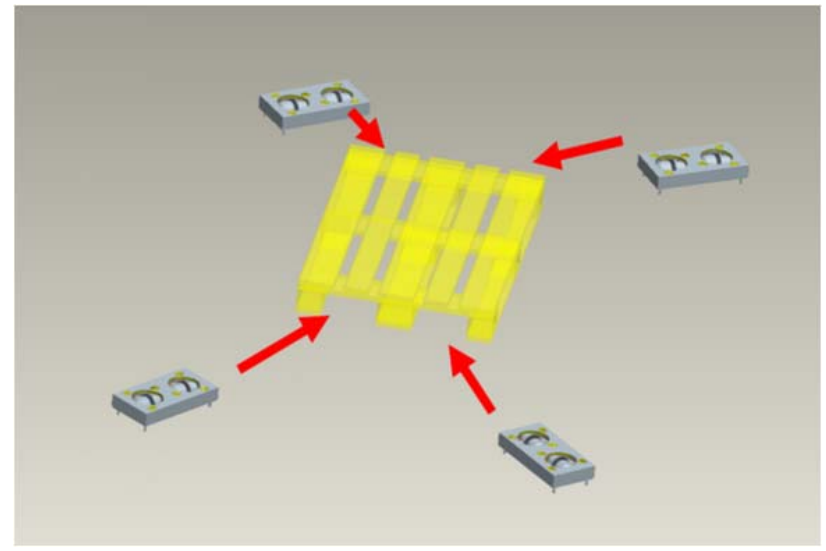

Abbildung 4. Verbund von 4 BInE-Modulen

\subsubsection{Multishuttle Move}

Mit dem Forschungs- und Entwicklungsprojekt „MultiShuttle Move” des Dortmunder FraunhoferInstituts für Materialfluss und Logistik (IML) wurde die Idee der Zellularen Transportsysteme in die Realität umgesetzt. Es wurde ein Fahrzeug für ein multimodales Logistikkonzept entwickelt, das den Anforderungen der Zellularen Transportsysteme entspricht und zusätzlich denen der Shuttle-Systeme gerecht wird. Da das neue Multishuttle Move über ein Boden- und ein Regalfahrwerk verfügt, kann es sich sowohl in einem Regallager wie ein konventionelles Shuttle als auch auf dem Lagerboden ähnlich wie ein Fahrerloses Transportfahrzeug bewegen (vgl. Abbildung 5).

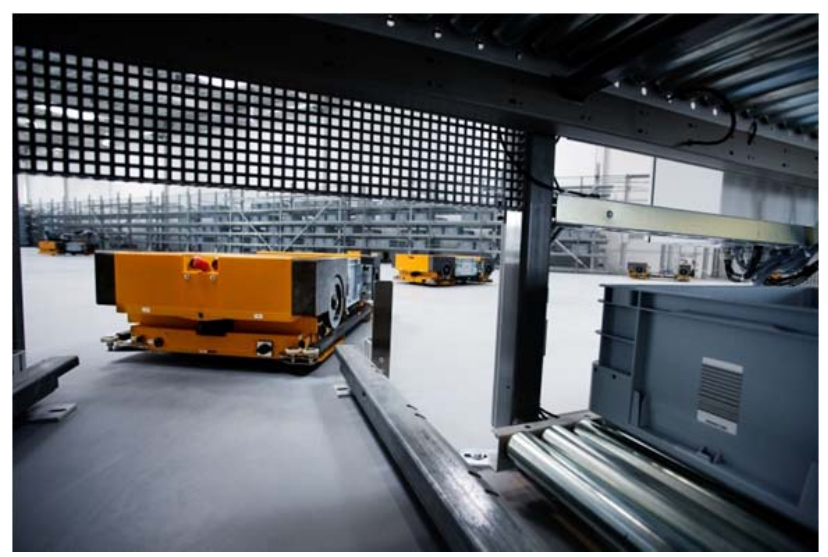

Abbildung 5. Multishuttle Move

Das Fahrzeug kann Kleinladungsträger $(600 \mathrm{~mm} \times$ $400 \mathrm{~mm}$ ) bis zu einem Gewicht von $40 \mathrm{~kg}$ aufnehmen. Es bewegt sich mit einer Geschwindigkeit von $1,0 \mathrm{~m} / \mathrm{s}$ auf dem Boden und mit Geschwindigkeiten bis 2,0 m/s im Regallager und wiegt dabei kaum mehr als das regalgebundene Multishuttle. Das modulare Energiekonzept mit den momentan eingebauten Akkumulatoren ermöglicht einen durchgehenden Betrieb von mehr als 4,5 h ohne Nachladen. Die intelligente Lokalisation, Navigation und Kollisionsvermeidung basiert auf dem neu entwickelten hybriden Sensorikkonzept, bestehend aus Odometrie, Funkortung sowie Abstands- und Inertialsensoren.

Das Fahrzeug kann sein Wissen mit dem Schwarm teilen und bewegt sich völlig frei auf der Fläche. Es sucht sich jeweils die kürzeste Route zum Ziel - dadurch wird ein optimaler Durchsatz gewährleistet.

\subsection{LOW-COST-FAHRZEUGE}

Aktuell existieren weitere Trends, die sich in den Bereich der Realisierung kleinskaliger, sehr kostengünstiger und wenig intelligenter Fahrzeuge einordnen lassen. Diese lassen sich nicht direkt zu Zellularen Transportsystemen einordnen, da diesen Systemen die Intelligenz fehlt autonom zu handeln oder mit anderen Systemen zu interagieren. Jedoch liegt die Vermutung nahe, dass in naher $\mathrm{Zu}-$ kunft durch mehr Rechenleistung diese Systeme mehr Autonomie gewinnen und sich zu den Zellularen Transportsystemen einordnen lassen.

Beispiele für die Entwicklungen sind das Projekt „Kleine automatische Transporteinheit - KATE“ der Universität Stuttgart und des Unternehmens Götting (siehe Abbildung 6) sowie das Projekt „Low Cost Automated Guided Vehicle - LOCATIVE“ des Fraunhofer IML (siehe Abbildung 7).

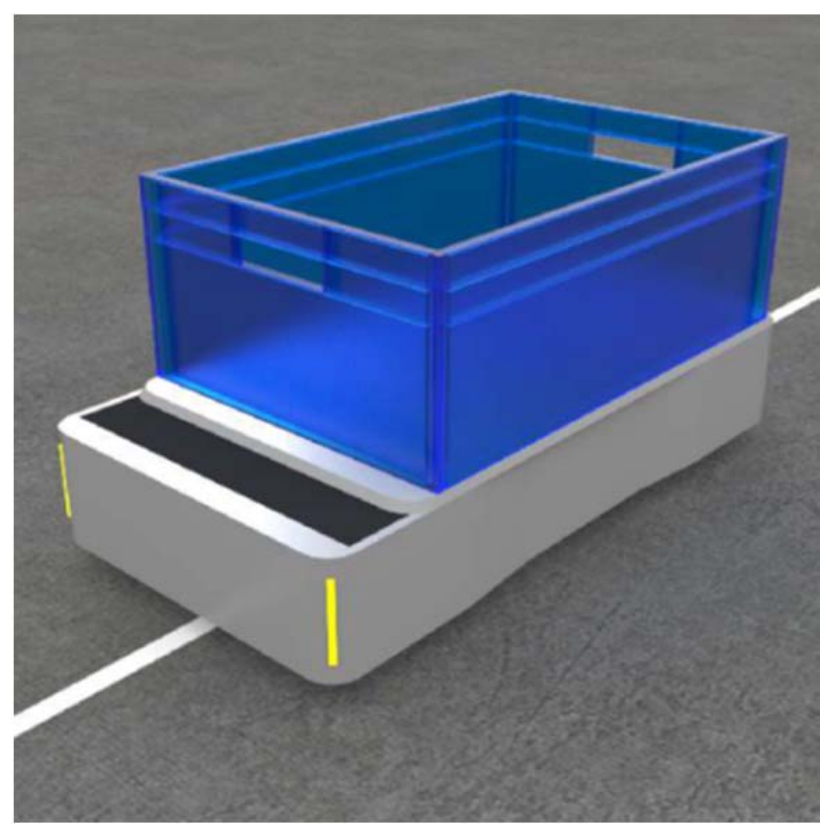

Abbildung 6. KATE-Fahrzeugplattform 


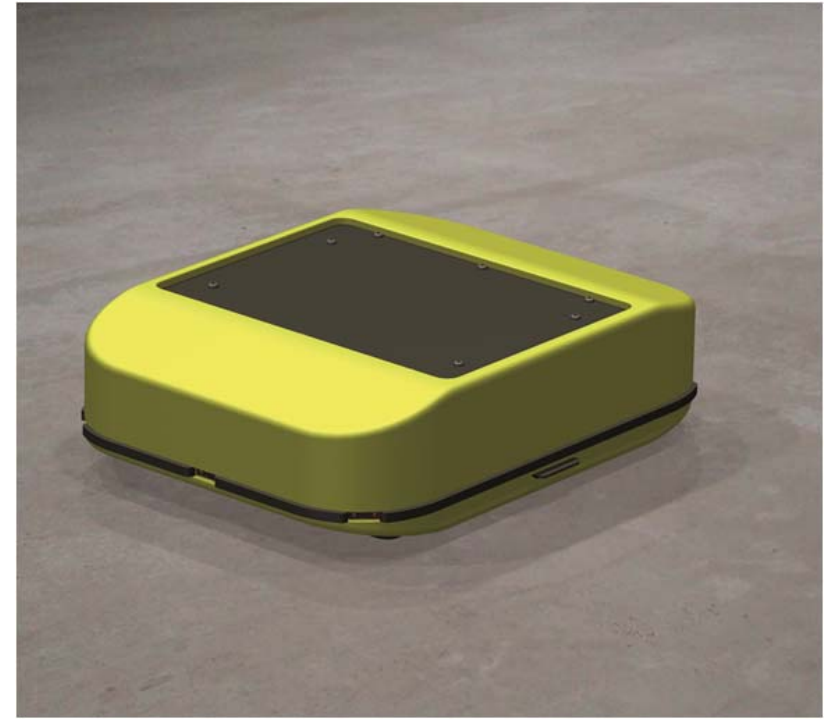

Abbildung 7. LOCATIVE

\subsection{FORSCHUNGSSCHWERPUNKTE DES FRAUNHOFER IML}

\subsubsection{ZELLULARE TRANSPORTSYSTEME}

Zellulare Transportsysteme weisen im Bereich der Topologieflexibilität gegenüber den Stetigfördersystemen Vorteile auf, da sie in der Lage sind, eigenständig ohne manuelle Eingriffe die Topologie an die neuen Gegebenheiten anzupassen. Zellulare Transportfahrzeuge basieren auf autonom fahrenden Transportentitäten. Dies sind autonome Transportfahrzeuge wie Shuttles oder intelligente Fahrerlose Transportfahrzeuge. Die Kommunikation der Entitäten untereinander erfolgt, wie auch die Steuerung selbst, mit einer dezentralen Architektur (z. B. durch ein Multi-Agenten-System). Zellulare Transportfahrzeuge sind topologieflexibel, d. h. die Anordnung der Transportentitäten im Raum (das Streckennetz bzw. Layout) kann jederzeit manuell geändert oder durch das System selbständig geändert werden. Zellulare Transportfahrzeuge sind intralogistische Systeme höchster Flexibilität [tHK11; KSN11].

\subsubsection{SCHWARMINTELLIGENZ}

Schwarmintelligenz in künstlichen Systemen vereint mehrere Individuen, die mit einer dezentralen Steuerung und Selbststeuerung agieren. Sie konzentriert sich auf das Verhalten des Kollektivs, welches von der Interaktion mit Individuen untereinander und mit der Umwelt profitiert.

Ähnlich wie in natürlich vorkommenden Schwärmen (z. B. Fischschwarm) wird keine zentrale Instanz zur Steuerung der Individuen benötigt, so dass die Aufgabenentgegennahme und -organisierung dezentral verwaltet werden kann [DBT99].

\subsubsection{SENSORFUSION}

Der Hauptzweck der Sensorfusion ist es genauere Informationen aus seiner Mehrzahl von Sensoren durch probabilistische Ansätze als durch einzelne Auswertung der Sensoren zu gewinnen. So kann durch Sensorfusion die Anzahl der verwendeten Sensoren reduziert werden oder die Messgenauigkeit erhöht werden. Dies kann mit Fuzzy Logic, Kalman-, Extended Kalman- oder Partikelfiltern erreicht werden. Für gewöhnlich wird dafür ein Sensormodel, speziell für prädiktive Algorithmen, verwendet. So werden Sensorfusionsalgorithmen für die Lokalisierung (vgl. Abbildung 8), Kollisionsvermeidung und Objektklassifikation bei Zellularen Transportsystemen eingesetzt.

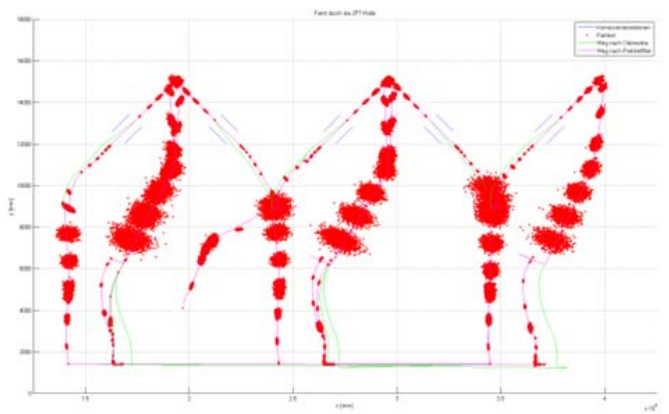

Abbildung 8. Fusionierte Positionsdaten eines MS Move

\subsubsection{Multi-AgENTEN-SYSTEM}

Das Hauptziel von Multiagentensystemen ist es komplexe Systeme ohne eine zentralen Entität zu steuern. Diese Systeme arbeiten mit einer Menge von Agenten die autonom, proaktiv, adaptiv und reaktiv sind. Softwareagenten besitzen spezielle Fähigkeiten um Probleme zu lösen, Informationen zu sammeln, Konflikten vorzubeugen und mit anderen Agenten kommunizieren zu können um ein übergeordnetes Problem zu lösen [Gue04; Zub10].

\subsection{SYSTEMARCHITEKTUR}

Das Ziel ist die Gestaltung eines anpassbaren, flexiblen und einfach zu skalierenden Intralogistiksystems. Die „Multishuttle Move“-Architektur ist nachfolgend beschrieben und stellt die Realisierung am Fraunhofer IML dar.

Die Steuerung des Multishuttle Move lässt sich in drei Ebenen unterteilen (vgl. Abbildung 9) [KG11; KA11]:

- $\quad$ Sensor- und Aktorebene

- Operative Ebene

- $\quad$ Strategische Ebene oder auch Schwarmebene. 


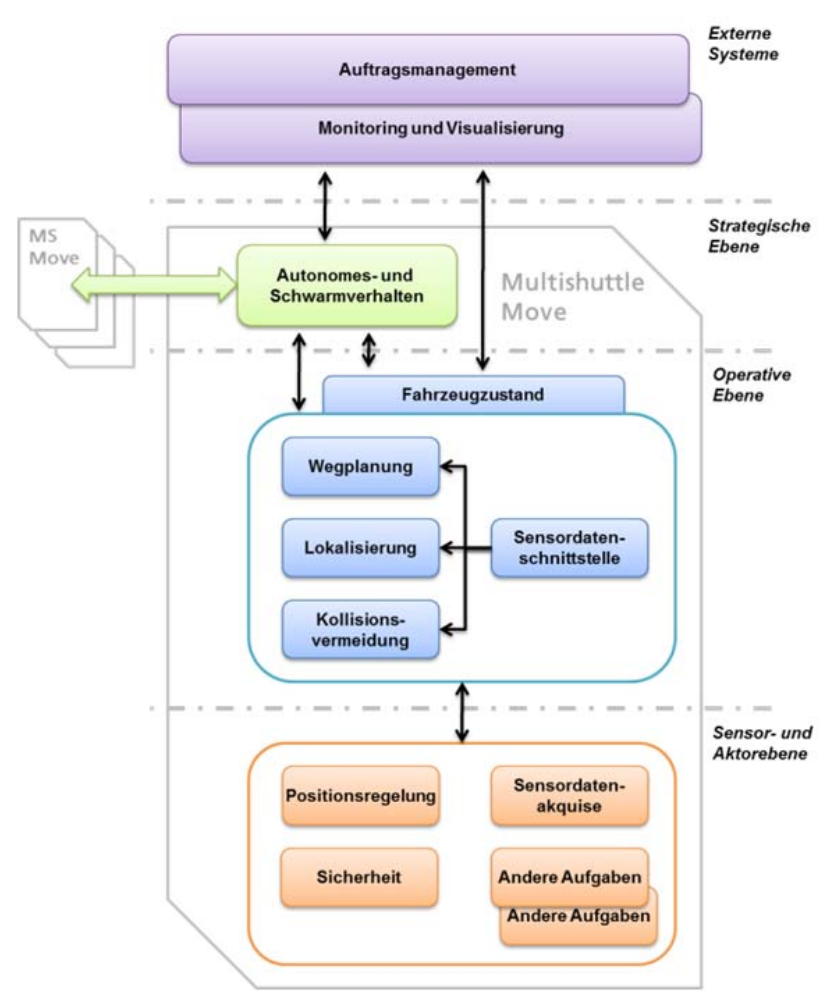

Abbildung 9. Steuerungsarchitektur MS Move

Auf der Sensor- und Aktorebene werden zeitkritische Aufgaben, wie Positionieren, Bedienung des Lastaufnahmemittels, Akquise der Sensordaten und allgemeine Steuerungsaufgaben, auf unterster Ebene hardwarenah abgearbeitet. Auch alle sicherheitskritischen Aufgaben, wie der Kollisionsschutz (Performance Level d nach EN ISO 13.849 und SIL2 nach IEC 61.508). Die Realisierung dieser Ebene erfolgt mittels einer Soft-SPS, die auf dem Industrie-PC (IPC) parallel zu den nachfolgen Tasks läuft.

Die operative Ebene ist wesentlich komplexer, und beschäftigt sich mit weniger zeitkritischen Aufgaben wie mit der intelligenten Auswertung der Daten der Funkortung sowie der Abstands- und Inertialsensoren. Die Kollisionsvermeidung und die Lokalisation sind ebenfalls Bestandteile dieses Layers. Dort wird ebenso die aktuelle Trajektorie des Fahrzeugs abhängig von der aktuellen Situation berechnet. In dieser Ebene werden auch die Informationen für die Schwarmebene wie die aktuelle Position, der Ladungszustand, die aktuelle Geschwindigkeit etc. der Schwarmebene bereitgestellt. All diese Tasks sind als einzelne Threads auf dem Betriebssystem des IPC realisiert.

Einen direkten Einfluss auf die operative Ebene übt die strategische Ebene aus. Letztere akquiriert die bereitgestellten Informationen über den aktuellen Zustand des Fahrzeugs und fällt Entscheidungen, die für die Funktion des Fahrzeugs als Individuum und Teil eines Schwarms notwendig sind. Ein Software-Agent steuert dynamisch die Parameter der operativen Ebene, abhängig von seinem aktuellen Auftrag und den Aufträgen der anderen Fahr- zeuge. Die strategische Ebene, auch Schwarmebene genannt, ist an externe Systeme wie das Warehouse Management System (WMS) oder sogar direkt an das Enterprise Resource Planning (ERP) angebunden.

Exemplarisch ist hier ein Ablauf dargestellt: Durch das WMS werden Transportaufträge generiert und in einem Auftragspool bereitgestellt. Die Software-Agenten der einzelnen Fahrzeuge bewerben sich mittels eines Biete-Algorithmus um die einzelnen Aufträge. Dabei werden Auftragseigenschaften wie Sequenz und Ziel sowie der Fahrzeugzustand vom Agenten berücksichtigt. Nach der erfolgreichen Gebotsabgabe führt das Zellulare Transportsystem die Aufträge aus.

\subsection{TeChNische ReALISIERUNG}

Das MS Move integriert alle genannten Funktionalitäten: Sensorik, Aktorik und Kommunikation. Smart Sensors und Algorithmen der Sensorfusion für die Navigation, Lokalisierung und Kollisionsvermeidung befähigen das MS Move seinen Zustand zu erfassen und zu analysieren. Um die Multisensor- und Multisystem-Kommunikation zu realisieren, wird für dieses Projekt momentan eine modulare Softwareplattform Unified Sensor Data Interface (USDI) entwickelt. Durch die Verwendung von standardisierten Kommunikationsprotokollen wie TCP/ IP und UDP wird eine volle Kompatibilität zu bestehenden Systemen sichergestellt. Es gewährt Zugriff auf alle angeschlossenen Sensoren über Sockets ohne ihre physikalische Anbindung und Position zu kennen. Jedes plattformunabhängige Softwaremodul des USDI beschreibt einen Sensor und kann von dem gesamten Netzwerk aus über einen vereinheitlichten Datenstrom - Unified Sensor Data Protocol (USDP) - zugänglich gemacht werden. Zusätzliche Informationen wie Sensortyp, Messwertdimension und Datenlängen werden bereitgestellt. Daten von virtuellen und simulierten/ emulierten Sensoren wie auch fahrzeugspezifische Informationen können ebenfalls kommuniziert werden.

Ein Teilnehmer kann Datenkanäle erstellen, die exklusiv für ihn oder öffentlich zugänglich sind. Diese Schnittstelle ermöglicht so heterogene Sensorfusion zur Navigation und Sicherheitsbelange in Echtzeit. In dem MS Move werden die Messdaten von vier verschiedenen Arten von Sensoren fusioniert: Sicherheitslaserscanner, die auch zum Kollisionsschutz verwendet werden, drahtlose Sensorknoten, die über Ankerknoten Entfernungsinformationen generieren (IEEE 802.15.4 Standard), Inertialsensoren und Odometriesensoren des Differentialantriebes. Die Round-Trip-Time-of-Flight-Messung basiert auf einem Sensorknotennetzwerk, die als Real-Time Lokalisierungssystem (RTLS) arbeiten [KR10]. Ein mobiler Marker in diesem RTLS lokalisiert sich durch Entfernungsmessung zu einer Menge von Ankerknoten als Referenzpunkte. Die Ankerknoten sind in einem kartesischen Koordinatensystem an definierten Positionen angebracht. 
Dadurch kann mit Hilfe von Trilateration die Position des mobilen Markers berechnet werden. Für eine eindeutige Lokalisierung werden mindestens 3 Ankerknoten benötigt. Die Genauigkeit des Round-Trip Time-of-Flight wird durch die Anwendung eines Extended-Kalman-Filters (EKF) auf die Entfernungsmesswerte verbessert. Die Implementierung wird in Röhrig et al. [RM09] beschrieben.

\subsubsection{NAVIGATION UND LOKALISIERUNG}

Die Lokalisierung des Fahrzeugs in der Schiene wird mit Koppelnavigation und mit Hilfe von Lichtschranken, die die Fachschlitze der Multifunktionsschiene erkennen, erreicht. Außerhalb der Schiene ist die Lokalisierung komplexer. Mehrere Messdaten verschiedener Sensortypen werden hier fusioniert, um eine Genauigkeit von wenigen Millimetern zu erreichen. Der Lokalisierungsalgorithmus wird als Monte-Carlo-Filter (MPF) basierend auf Bayes'schen Modellen implementiert und verwendet Koppelnavigation in der Prädiktionsphase, Messwerte der Laserscanner in der Updatephase, basierend auf dem Ansatz wie in Thrun et al. [TBF05]. Dieser Algorithmus kann sowohl auf lokale als auch auf globale Lokalisierungsprobleme angewendet werden. Durch den modularen Aufbau des Lokalisierungsalgorithmus ist es möglich die bestehende Sensorik für zukünftige Anwendung durch andere zu ersetzen und dabei nur die Sensormodelle auszutauschen (z. B. Wechsel der Laserscanner durch 3DSensoren).



Abbildung 10. Flussdiagramm zum MCP

\subsubsection{ENERGIEEFFIZIENZKONZEPT}

Ein weiteres Schlüsselelement für die erfolgreiche Umsetzung des Schwarmgedanken ist das adaptive Batteriekonzept, welches auf mehreren Anforderungen beruht. Die Fahrzeuge arbeiten mit Akkumulatoren als Hauptenergiequelle und werden in Stromschienen des Hochregallagers nachgeladen. Das FTF basiert auf einem gewichtsoptimierten Design und verwendet energiesparende Komponenten, wie einen Industrie-PC, Leistungselektronik für die drei bürstenlosen Antriebe für den Antrieb und das Lastaufnahmemittel. Zusätzlich kann die Bremsenergie mit geringen Verlusten in die Energieversorgung zu- rückgespeist werden. Des Weiteren ist ein adaptives Energiekonzept im MS Move implementiert - jegliche Energiequelle wie LiIon-, LiPoly-, Blei-Gel-Batterien oder Doppelschichtkondensatoren können im System eingebunden werden. Die Vorhersage für den Energieverbrauch für die Fahrstrecke und ein hochintegriertes Energiemanagement sind Hauptbestandteile der Forschung. Die Untersuchung der Energieversorgung fokussiert sich hauptsächlich auf Konzepte wie Energierückgewinnung, energieeffiziente Wegplanung und optimierte Motorsteuerung [KKtH10]. Ein durchdringendes Energiekonzept ist Wegbereiter für batteriegetriebene Fahrzeuge.

\section{ERSTE ERFAHRUNGSWERTE}

Den großen Praxistest absolviert das Gesamtsystem zurzeit im Dortmunder Fraunhofer IML seit Juni 2011. In einer eigens dafür gebauten, $1.000 \mathrm{~m}^{2}$ großen und $65 \mathrm{~m}$ langen Versuchshalle übernimmt ein Schwarm von 50 Multishuttle Move die Aufgaben klassischer Materialflusstechnik (siehe Abbildung 11). Der Betrieb wurde bereits kurz nach der Eröffnung realisiert.

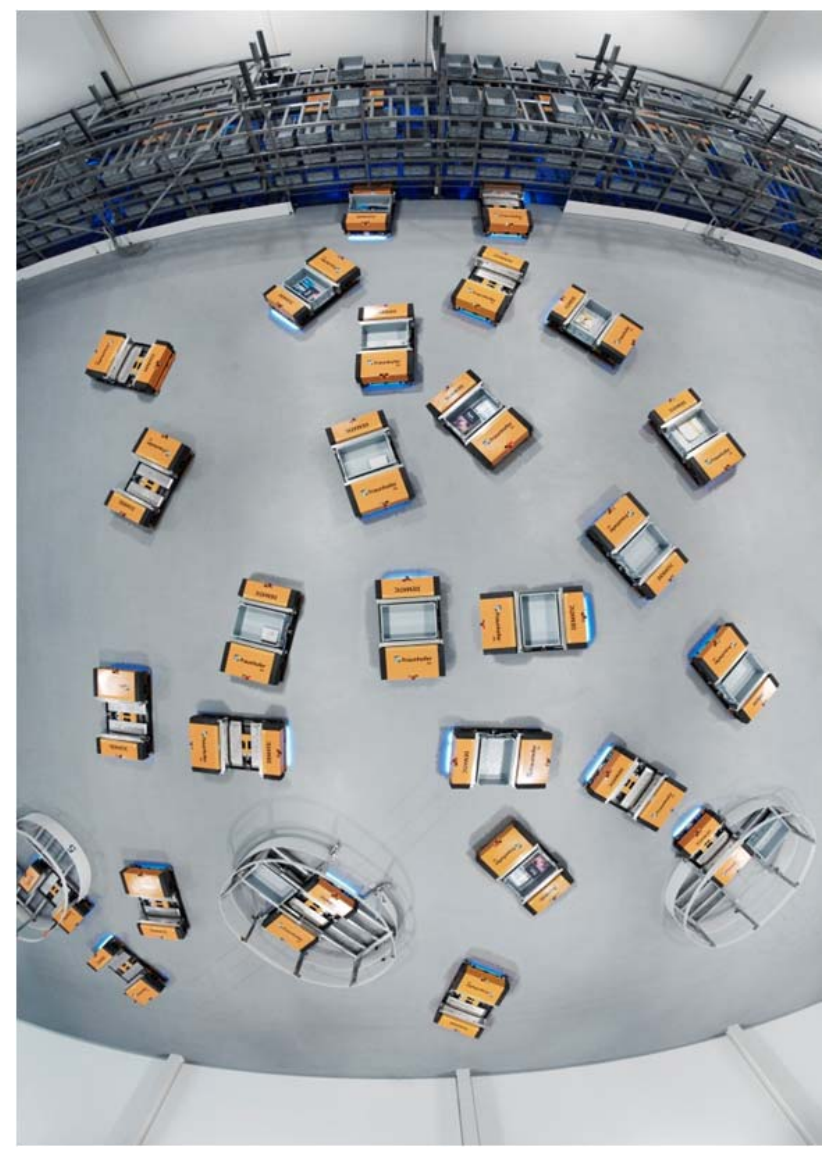

Abbildung 11. Multishuttle Move in der Versuchshalle am Fraunhofer IML in Dortmund 
Die gesamte Anlage befindet sich in einem funktionsund einsatzfähigen Stadium, was bereits bei zahlreichen Demonstrationen, unter anderem auf dem Zukunftskongress der Logistik 2011, gezeigt wurde. Die Erfahrungen zeigen, dass Herausforderungen für die Forschung im Bereich des Schwarmverhaltens und der Sensorik existieren. Im Bereich des Schwarmverhaltens werden aktuell neue Steuerungsstrategien für den Fahrzeugschwarm erforscht, um einen Zuwachs der Transportleistung mit mehr als 33 Fahrzeugen im aktuellen Layout nachzuweisen. Die Sensorikforschung wird aktuell in Zusammenarbeit mit universitären Einrichtungen sowie Sensorikherstellern, wie den Unternehmen SICK AG sowie ifm electronic GmbH in einem Praxistest untersucht, so dass auch in Zukunft weitere, verbesserte Schwarmfahrzeuge in Dortmund am Fraunhofer IML gebaut werden.

\section{Ausblick}

Gegenwärtig ist der getätigte Invest für die Vergrößerung eines Materialflusssystems basierend auf Zellularen Transportsystemen proportional zum Durchsatz, ohne zusätzliche Kosten für die Anpassung an das bestehende System (vgl. Abbildung 12). Das bedeutet, dass sich der Maximaldurchsatz am benötigten Durchsatz orientiert. Durch ihre Flexibilität haben Zellulare Transportsysteme ökonomische Vorzüge im Vergleich zu konventioneller Fördertechnik, da diese für kommende Anforderungen oder Hochlastzeiten überdimensioniert werden müssen. In Bezug auf Flexibilität und Wandelbarkeit hat ein ZTS Vorteile gegenüber herkömmlicher Fördertechnik. Ökonomische Aspekte (Wie viel kostet Autonomie?) und funktionale Aspekte (Wie viel Autonomie wird benötigt?) setzen die Grenzen für den Schwarm und den Grad der Autonomie.



(a)

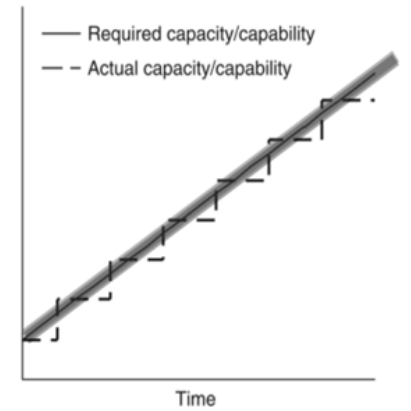

(b)
Abbildung 12. Optimale Anpassung eines Materialflusssystems. (a) Unflexibles System, (b) Flexibles System, (Grau in b) ZTS [NtH10]

Diese Aspekte sind Schwerpunkte weiterer Forschungsaktivitäten. Für singulär benötigte Rechenleistung wird in Betracht gezogen, komplexe Steuerungsaufgaben in die Cloud auszulagern. Zur Balancierung der benötigten Intelligenz in einem komplexen System sowie Erhöhung des Dezentralisierungsgrads kann die Verteilung der
Steuerungsaufgaben in der Zellularen Intralogistik direkt auf das Stückgut mit Hilfe von intelligenten Behältern wie InBins ${ }^{\circledR}$ erfolgen. Dadurch kann die maximale Flexibilität und Skalierbarkeit gewährleistet werden.

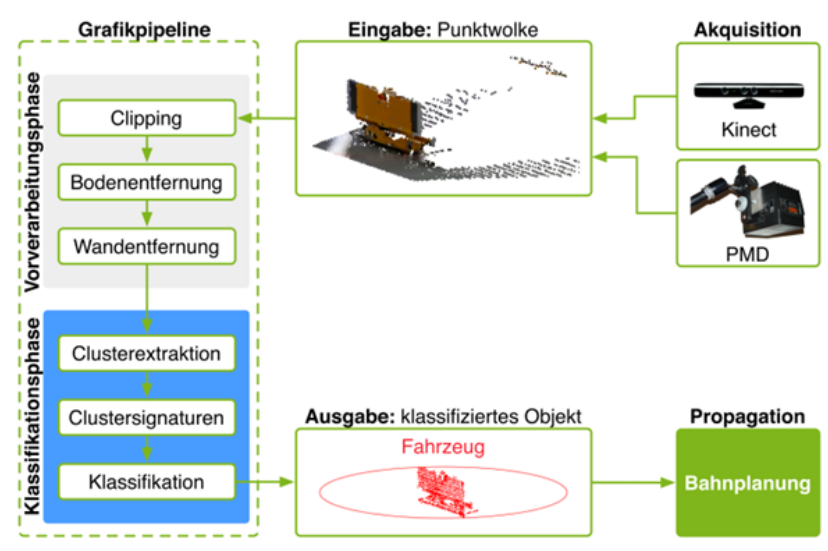

Abbildung 13. Klassifikation eines MS Move mittels 3D-Sensorik

Ein weiteres Problem in der Intralogistik besteht in der homogenen und wenig strukturierten Umgebung. Diese bietet wenig unterscheidbare Merkmale für die Lokalisierung und Kollisionspotentiale mit Messsensorik. Aus diesem Grund zukünftig 3D-Sensoren eingesetzt werden (siehe Abbildung 13), die zum Kollisionsschutz und zur Verringerung der Gefahren für Menschen und Maschinen beitragen sollen (siehe Abbildung 14).

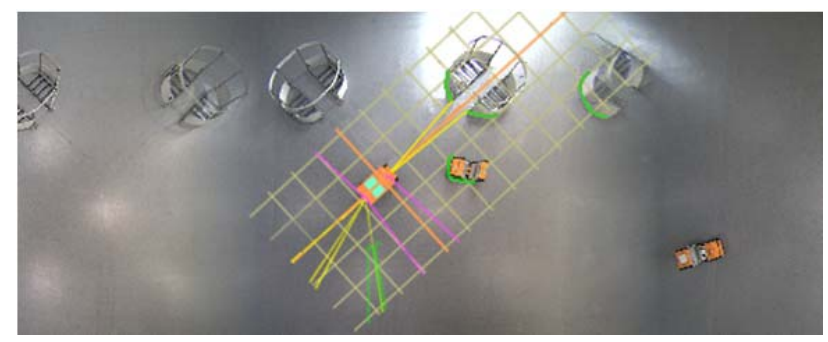

Abbildung 14. Anfahrt des MS Move an eine Arbeitsstation

\section{LITERATUR}

[AW08]

D’Andrea, Raffaello; Wurman, P.: Future challenges of coordinating hundreds of autonomous vehicles in distribution facilities. In: Technologies for Practical Robot Applications, 2008

[AWM08] D’Andrea, Raffaello; Wurman, P.; Mountz, M.: Coordinating hundreds of cooperative, autonomous vehicles in warehouses. In: AI Magazine, Band 29, Nr. 1, 2008, S. 9-19 
[BSS08]

Baur, Tobias; Schönung, Frank; Stoll, Thomas; Furmans, Kai: Formationsfahrt von mobilen, autonomen und kooperierenden Materialflusselementen zum Transport eines Ladungstragers. In: Tagungsband zum 4. Fachkolloquium der Wissenschaftlichen Gesellschaft für Technische Logistik, Band 4, 2008

[DBT99] Dorigo, Marco; Bonabeau, E.; Theraulaz, G.: Swarm Intelligence From Natural to Artificial Systems. Oxford University Press, 1999

[GtH10] Günthner, Willibald. A.; ten Hompel, M.: Internet der Dinge in der Intralogistik. Berlin, Heidelberg: Springer-Verlag, 2010

[Gue04] Guessoum, Zahia: Adaptive Agents and Multiagent Systems. IEEE distributes systems online, Band 5, Nr. 7, 2004

[Gui08] Guizzo, Erico: Three engineers, hundreds of robots, one warehouse. IEEE Spectrum, Band 45, Ausg. 7, 2008, S. 26-34

[HFS09] Hippenmeyer, Heinrich; Furmans, Kai; Stoll, Thomas; Schönung, Frank: Ein neuartiges Element für zukünftige Materialflusssysteme: KARIS - dezentral gesteuert. In: Hebezeuge Fördermittel, Band 49, Nr. 6, 2009, S. 304-306

[tHK11] ten Hompel, Michael; Kamagaew, Andreas: Zellulare Intralogistik - in $\mathrm{Zu}$ kunft nur noch Multishuttles?. In: 20. Deutscher Materialflusskongress, München, 2011

[KA11] Kamagaew, Andreas; Albrecht, Thomas: Fortschritt durch Wandel. In: Logistik heute, Band 4, S. 54-55, 2011

[KG11] Kamagaew, Andreas; Große, Eric: Zellulare Transportsysteme - Multishuttle Move, Multimodales Intralogistikkonzept. Hebezeuge Fördermittel, Band. 51, Nr. 4, S. 170-172, 2011

[KKtH10] Kamagaew, Andreas; Kirks, Thomas; ten Hompel, Michael: Closed Loop Velocity Control for an AGV Equipped with a Modified Voith-Schneider-Drive. In: Symposium Gyro Technology, Karlsruhe, 2010
[KR10]

[May09]

[NtH10]

[RMT07]

[SRS07]

[TBF05]

[Zub10] Zuber-Knost, Elisabeth: Von Ameisen lernen. Karlsruher Institut für Technologie - Presseinformation, Band 64, 2010 